\title{
Particles of man (part II)
}

\author{
Giulio Maltese
}

Published online: 12 March 2014

(c) Centro P.RI.ST.EM, Università Commerciale Luigi Bocconi 2014

\begin{abstract}
This article-the second of a three-part series-aims to briefly retrace the milestones in the development of particle physics, which led to what we believe is a consolidated framework, manufactured by man to explain what he himself observes, in his role as part of nature.
\end{abstract}

Keywords History of particle physics · Quantum mechanics · P. A. M. Dirac · Quantum electrodynamics . Enrico Fermi · Beta decay · Strong interactions · Weak interactions · Isotopic spin · Pion · Muon · Strange particles $\cdot$ Hadrons $\cdot$ Eugene Wigner

This article is the second of a three-part series that briefly retraces the milestones in the development of particle physics, which led to what we believe is a consolidated framework, manufactured by man to explain what he himself observes, in his role as part of nature. See Part I in Lettera Matematica International Edition, Lett Mat Int (2013) 1:145-154 (DOI 10.1007/s40329-013-0025-x).

\section{Weak interactions do not preserve the parity}

If the proton-neutron model of Heisenberg represented the first step towards the way we look at the atomic nucleus today, Fermi's theory certainly opened the path leading to the modern electroweak theory $(\S 10)$. The weak force is somehow "different" from the other interactions. In

G. Maltese ( $\square)$

Via Tiberio Imperatore 65, 00145 Rome, Italy

e-mail: giulio_maltese@libero.it contrast to the electromagnetic, strong and gravitational cases, we do not know of physical systems with entangled states arising from weak interactions. The latter play a crucial role in radioactive beta decay, and more generally in the decay of quarks and leptons $(\S 10)$ and at the cosmic level. For example, the nuclear chain reactions inside the sun begin and depend in a crucial manner on the reaction $p+p \rightarrow{ }^{2} \mathrm{H}+e^{+}+v e$ caused by the weak force. ${ }^{1}$ The average life of particles whose decay depends on the weak force is typically of the order of $10^{-10} \mathrm{~s}$, relatively long if compared with the electromagnetic $10^{-19} \mathrm{~s}$ and the strong interaction's $10^{-23} \mathrm{~s}$.

In Fermi's theory the adimensional constant $\alpha W$ associated to the force's intensity equals $\left(m p c^{2}\right)^{2} G F /(\hbar c)^{3}$, where $G F$ is the Fermi constant $\left(G F /(\hbar c)^{3}=1.1664 \times 10^{-5}\right.$ $\mathrm{GeV}^{-2}$ ) and $m p$ is the mass of the proton. The value of $\alpha W$ is $1.027 \times 10^{-5}$, that is to say, three orders of magnitude smaller than $\alpha E M$. So just as $\alpha E M$ is proportional to the square of the elementary electric charge $e$, for the weak interaction we can define a relation $\alpha W=g W^{2}$, where $g W$ can be thought of as a weak charge, the weak analogue of the electric charge. Fermi's theory, although still now a cornerstone, has been modified over time to adapt to the new observations and discoveries. Already in 1936, for instance, George Gamow and Edward Teller noticed that the theory wasn't able to account for the beta decay of nuclei with spin that jumped by 1 , while it explained well the transitions between nuclei with the same spin.

Bruno Pontecorvo, Giampietro Puppi and others, motivated by the result obtained in Rome by Conversi, Pancini and Piccioni, showed in 1947 that the beta decay, the muon

\footnotetext{
${ }^{12} \mathrm{H}$ denotes the deuteron, the nucleus of the hydrogen isotope (called deuterium) with mass number $A=2$, discovered in 1932. The deuteron consists of one proton and one neutron.
} 
decay and the capture of negative muons observed in Rome could be all interpreted as processes caused by a unique weak interaction, for they had similar values of the coupling constant. The idea of the universality of this interaction thus arose, and resisted for many years to come until the introduction of the Cabibbo angle in 1963.

In 1956, using the antineutrinos produced at the Savannah River reactor, Frederick Reines (Nobel Prize for Physics in 1995) and Clyde Cowan proved the reaction $\bar{v}_{e}+p \rightarrow n+e^{+}$(the inverse beta decay, in which an antineutrino hits a proton and generates a neutron and a positron). This was the first clear signal of the existence of the neutrino. The authors sent a telegraph message to Pauli, who celebrated with some friends and a case of champagne.

Precisely at this time a fundamental discovery was made. At the beginning of the 1950s no one doubted the preservation of parity in all physical phenomena. To say that parity is preserved during a process is the same as accepting that the mirror image of a process is a phenomenon that can occur in nature. In studying the decay of strange particles, in particular those we now call $K$ mesons, scientists had observed a behaviour soon to be known as the " $\tau-\theta$ puzzle". Whilst observing the reactions $\theta^{+} \rightarrow \pi^{+}+\pi^{0}$ and $\tau^{+} \rightarrow \pi^{+}+\pi^{+}+\pi^{-}$it was noticed that the particles $\theta^{+}$and $\tau^{+}$had equal mass and comparable average life, so it was only natural to suppose they were the same particle. But if that were true, then there was a problem. The experimental data, in fact, strongly suggested that $\tau^{+}$had spin 0 and negative parity (the three pions in which it decayed had spin 0 and negative parity). Yet if $\theta^{+}$ and $\tau^{+}$were two decay modes of the same particle, then $\theta^{+}$ should have spin 0 and negative parity, too. And this could surely not be true, for the reaction $\theta^{+} \rightarrow \pi^{+}+\pi^{0}$ tells that if $\theta^{+}$had spin 0 it would have positive parity. So what do we do, then? A particle cannot have a parity that is sometimes positive and sometimes negative. Therefore, either $\theta^{+}, \tau^{+}$were distinct particles and so it was necessary to explain the equal masses and equal average lifetimes, or they were a single object, and there was something wrong in the very notion of the weak interaction governing their decay. The riddle was solved in 1956 by two very young former students of Fermi, Chen Ning Yang and Tsun Dao Lee. They analysed the conservation of parity during weak interactions and observed that there had never been an experiment confirming it (as opposed to strong and electromagnetic interactions, for which there was plenty of evidence supporting the preservation of parity). The $\tau-\theta$ puzzle could be sorted out simply by relinquishing parity conservation in weak processes. Lee and Yang proposed several experiments to test their conjecture. One in particular, carried out at the beginning of 1956, proved that parity is not preserved when $\mathrm{Co}^{60}$ nuclei, oriented by an external magnetic field, decay: Lee and Yang were right and shared the 1957 Nobel Prize. The subsequent development of their work showed that charge conjugation, too, which is a symmetry for both the strong and the electromagnetic force, is not preserved under weak interactions: the violation of parity exhibited by weak interactions was thus the sign of an asymmetry between the world of matter and that of antimatter (since conjugating the charge transforms a particle into its antiparticle).

The necessity of including the non-conservation of parity in weak interactions led in 1958 to an extension of Fermi's ideas, with the so-called $V-A$ theory ("Vector minus Axial") due to Richard Feynman and Murray GellMann, and, independently, Robert Marshak and Ennackal C. G. Sudarshan. Essentially, they added to the Hamiltonian some terms which are not invariant under spatial reflections. $^{2}$

For a few years after the discovery of Yang-Lee weak interactions were thought to violate $P$ and $C$ but still preserve the operation $C P$, in which, besides space reflections, every particle is replaced by its antiparticle. An experiment made in Brookhaven in 1964 proved, on the contrary, that also $C P$ could be violated in weak interactions, especially during the decay of $K^{0}$ mesons. The team conducting the experiment was headed by Val L. Fitch and James W. Cronin, one of the last students of Fermi: for that discovery they were awarded the 1980 Nobel Prize.

\section{The "Eightfold Way", the introduction of quarks and quantum chromodynamics}

The only hadrons known at the end of the 1940s were nucleons and pions. Starting from the 1950s, due to the employ of cosmic radiations and increasingly powerful accelerators, the number of hadrons grew massively, with

\footnotetext{
${ }^{2}$ Space reflections preserve the orientation of an axis (A), whereas polar vectors $(V)$ are reversed. The vector resulting from a cross product, e.g., the angular momentum, is a typical example of a pseudovector (also called an axial vector). Introducing in the Hamiltonian the term $(V-A) \cdot(V-A)$ gives rise to products $V A, A V$ that violate the parity $P$ and the charge conjugation $C$, despite preserving the symmetry $C P$. Fermi's theory is a theory of type $V-V$. An example of a quantity than changes under orientation reversal is the helicity $h=\mathbf{s} \cdot \mathbf{p} /(|\mathbf{s}||\mathbf{p}|)$, defined as the projection of the spin along the direction of motion. The numerator is the dot product of a pseudovector (the spin) with a polar vector (the linear momentum). Particles with spin in the direction of motion have right-handed helicity, equal +1 ; if the spin and the momentum are parallel but oppositely oriented, the particle is said to have helicity -1 or lefthanded. The first experiment conducted in 1958 and all subsequent tests have shown that the neutrino is left-handed and the antineutrino right-handed, always: to account for this fact scientists elaborated the so-called two-component theory of the neutrino.
} 


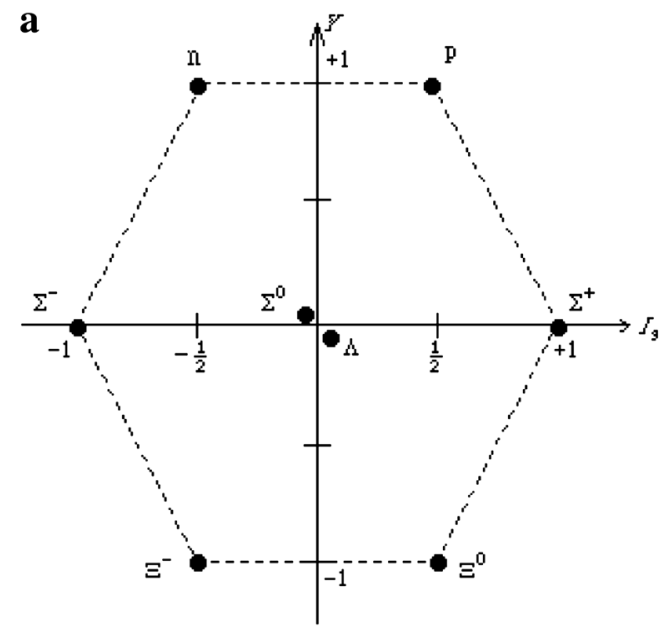

$\mathrm{N}(939)$

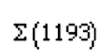

$\Lambda(1116)$

$\Xi(1318)$

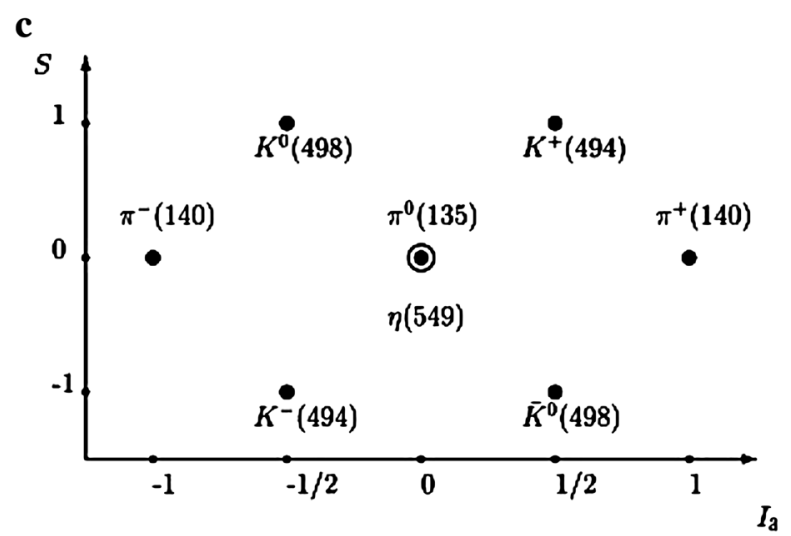

Fig. 1 a The baryon octet formed by particles with spin-parity $J^{P}=1 / 2^{+}$, represented on the hypercharge-isospin plane $\left(Y, I_{3}\right)$. Next to each multiplet is the average mass in $\mathrm{MeV} / \mathrm{c}^{2}$. Masses differ within each multiplet: for the neutron-proton doublet, for instance, the values are 938.27 (proton) and 939.57 (neutron). If the SU(3) symmetry were exact, the members of each multiplet would have the same mass (plus spin and parity). Instead the masses are slightly different, signalling the presence of some sort of symmetry break. In this case the different values of the mass depend on the electromagnetic force and the different masses of the constituent quarks. b The baryon decuplet formed by particles with spin-parity $J^{P}=3 / 2^{+}$. The top row is occupied by the so-called $\Delta^{++}$resonance, i.e., the first pion-nucleon resonance discovered and studied. Fermi was a central figure in the work that eventually led to its discovery, together with his Chicago

the discovery of strange particles, first, and then with the observation of several excited states of the known hadrons. Since strong interactions are invariant under rotations in the space of the isospin $I$, it was clear that the various charged states of hadrons (which formed an isospin multiplet) should have to be considered as different states of one particle, distinguished by the isospin component $I_{3}$. This begged the question of whether there existed other, broader schemes for classifying in a simpler way this larger

\section{b}
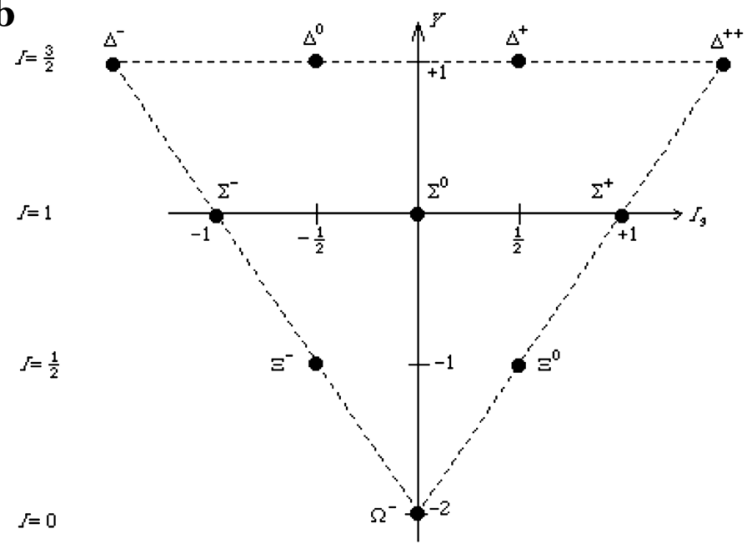

(1232)

(1384)

(1533)

(1672)

group (1951-1952). The "Delta" was the first in a series of baryon and mesonic resonant states whose symmetry properties paved the way to the introduction of quarks. It was the necessity to complete the decuplet, which only lacked the lower vertex, which led Gell-Mann to predict the existence of the $\Omega^{-}$particle (with its quantum numbers and mass) in 1962. The 1964 discovery of this particle in Brookhaven was one of the first results confirming the validity of Gell-Mann's scheme. c The mesonic octet with spin-parity $J^{P}=0^{-}$. the middle row consists of the three pions initially thought to carry the strong force $\left(I=1\right.$ and $I_{3}=-1,0,+1$ for $\left.\pi^{-}, \pi^{0}, \pi^{+}\right)$. Mesons have $B=0$ and $Y=S$. To these we should add the isospin singlet $\eta$ ' (958). The discovery of the $\eta$ and $\eta$ ' mesons (1962 and 1964) and of $\Omega^{-}$opened the door to the classification of mesons and baryons via SU(3)representations

number of particles. In 1961 Murray Gell-Mann (1969 Nobel laureate) and, independently, Yuval Ne'eman, developed a framework based on group theory.

They remarked that, within the approximations given by ignoring the difference of between the masses of hadrons with the same baryonic number, spin and parity, one obtained supermultiplets described by the representations of the Lie group SU(3). In fact, if we picture the elements of a supermultiplet as points on the $\left(I_{3}, Y\right)$-plane, we obtain 
structures like those of Fig. 1, which describes two representations of $\mathrm{SU}(3)$.

The eight generators of the group SU(3) proposed by Ne'eman and Gell-Mann were called by them the 'Eightfold Way', a reference to the core teachings of Buddhism. ${ }^{3}$ Today this group is called group of 'flavours' and denoted $\mathrm{SU}(3) f$, to distinguish it from the 'colour' group SU(3) we will see in a moment. The maximum number of commuting generators, and hence the number of generators represented by simultaneously diagonalisable matrices, is 2 , and these elements are related to the quantities $Y$ and $I_{3}$.

The discovery that hadrons could be classified as SU(3)supermultiplets may be compared to Mendeleev's discovery (1869) of the periodic table of elements: in both cases a certain phenomenological regularity was noticed, suggesting that the physical quantities of concern fitted into a classifying scheme, and in turn this scheme underlay their core structure and was related to the observed regularities. Since the representations of any group like SU(3) arise by suitably combining representations that in some sense have minimum dimension (here three), in 1964 Gell-Mann and George Zweig independently proposed assigning a physical meaning to states corresponding to 'minimal' SU(3)-representations. They assumed that hadrons were composed of three types of fundamental particles (and their antiparticles) having fractional electric charge: Gell-Mann called these basic objects quarks. ${ }^{4}$ Quarks accounting for the structure of nucleons (protons and neutrons) were named $u$ (up) and $d($ down); the quark present in strange particles became $s$ (from strangeness). Subsequently, to explain certain theoretical technical anomalies and the behaviour of recently discovered particles, further quarks were brought in. The new particles $c, b, t$ had quantum numbers related to flavour, and were called charm (conjectured in 1967 and detected in 1974 while studying the states of the massive meson $\psi=c \bar{c}$ ), ${ }^{5}$ beauty (discovered in 1977 by analysing the even more massive meson $\Upsilon=b \bar{b}$ ) and truth (1995) respectively. With the arrival of the additional flavours the

\footnotetext{
${ }^{3}$ Right View, Right Intention, Right Speech, Right Action, Right Livelihood, Right Effort, Right Mindfulness and Right Concentration form the "Noble Eightfold Way". Right View consists in understanding the Four Noble Truths: the truth of suffering, the truth of the origin of suffering, the truth of the cessation of suffering, the truth of the path leading to the cessation of suffering by means of the "Eightfold Way".

${ }^{4}$ Originating from $q u($ estion $\mathrm{m}$ )ark, the word was used for the first time in James Joyce's Finnegan's Wake (1939): “Three quarks for Muster Mark/Sure he hasn't got much of a bark/And sure any he has it's all beside the mark".

5 The particle $\psi$ was observed at the Stanford Linear Accelerator Center (SLAC) by the group headed by Burton Richter, and independently by the group of Samuel C. C. Ting in Brookhaven, who labelled it $J$. The new particle was thus called $J / \psi$ and its discoverers shared the 1976 Nobel Prize.
}

Gell-Mann/Nishijima relation was modified to $Q=I_{3}+(S+B+\mathrm{C}+\mathrm{B}+\mathrm{T}) / 2$, where $\mathrm{C}, \mathrm{B}$ and $\mathrm{T}$ are the quantum numbers of the flavours charm, beauty and truth. As for strangeness, also C, B and T are preserved by electromagnetic and strong interactions, and jump by one during weak processes.

The initial approach was to explain hadronic properties as entangled states of quarks (baryons consisting of three quarks, mesons of two quarks and one antiquark): for example the proton is made by the sequence $u u d$, the neutron by $d d u$ and the pion $\pi^{+}$by $u \bar{d}$, i.e., a pair $u$ anti$d$. It became clear very quickly that quarks should possess yet another internal quantum number, which was called 'colour'. This became necessary, to name but one of several reasons, so that the three quarks $u$ building the baryon $\Delta^{++}$, each with its own colour, satisfied Pauli's exclusion principle, despite their all having parallel spins and spacesymmetric wavefunctions. This new quantum number-the colour-must therefore take three values. Hadrons do not have a colour (colour has never been observed) even if the constituent quarks are coloured. This means the colours of the three quarks must somehow cancel each other out, giving 'zero' as a result. These considerations suggested the parallel to the colour palette, where three primary colours (e.g., red-green-blue) combine to produce no colour. Thus hadrons such as $\Delta^{++}$or $\Omega^{-}$are colourless, while the constituent quarks have a clear-cut colour. The analogy extends to mesons as well, which are formed by a quark-antiquark pair, because the colour of one quark neutralises the other one. Since the resulting hadrons are colour-neutral, only the combinations mentioned (three quarks or quark-antiquark pairs) are capable of generating entangled states, i.e., "white" hadrons.

At the end of the 1960s some physicists at the SLAC looked at electrons scattering through nucleons. The experiments were reminiscent of the behaviour of alpha particles beamed through nuclei observed at the beginning of the twentieth century by Hans Geiger and Ernest Marsden, and later interpreted by Rutherford as evidence for the existence of the atomic nucleus. In that case the surprise was that many more particles than expected were being deviated. The SLAC findings showed that nucleons similarly possessed a finer inner structure: again, the number of strongly deviated electrons was larger than the predicted value, a hint at the presence of infinitesimal "cores" of matter inside nucleons. One member of the SLAC team was Fermi's student Jerome I. Friedman, who went on to win the Nobel Prize in 1990 for this discovery. In 1969 Feynman and James D. Bjorken computed the expected distribution of scattered electrons that hit pointparticles: their predictions, derived from the so-called parton model, agreed with the experiments. Historically speaking, this represents the birth (albeit not the direct 
observation) of quarks, and the experimental proof that quarks were more than just the ingredients of a hypothetical mathematical model.

The idea underpinning the very notion of quark prompted Frank Wilczek and David J. Gross to create in the early 1970s quantum chromodynamics (QCD), the modern theory of strong interactions. QCD is a non-Abelian quantum field theory à la Yang-Mills: it is a gaugeinvariant and renormalisable theory based on the colour symmetry $\mathrm{SU}(3) c$, the matrix group acting on the three colours of quarks. The source of the strong force is the colour charge, and comes in three types: red, blue and green for quarks and antired, antiblue, antigreen for antiquarks. Each flavour of a quark, for instance $u$, can have any of the three colours, and $\mathrm{SU}(3) c$ acts on this triple of states. Interactions are mediated by gluons (from the word 'glue'), an octet of colour-charged particles with spin 1, negative parity and zero mass. The lack of mass is due to the conservation of gauge symmetry in QCD (by contrast, the flavour symmetry SU(3)f, originally defined by GellMann and Ne'eman, is broken, since the masses of quarks are different; for example $s$ is heavier than $u$ and $d$ ). Each gluon carries a colour and an anticolour charge, e.g., $r \bar{b}$, red-antiblue. The colour symmetry should be understood as follows: if every red quark became green, every green one turned blue and all blue ones became red, then all hadrons would stay colour-neutral. The symmetry is such that an overall colour change of the type described cannot be observed.

Gluons correspond precisely to the generators of the group $\mathrm{SU}(3) c$. Note that whereas photons, the mediators of the electromagnetic interaction, have no electric charge, gluons do have a colour charge and can thus interact with each other. The direct experimental confirmation of the existence of gluons came in the 1970s at Hamburg's PETRA (Positron Electron Tandem Ring Accelerator) during the study of high-energy electron-positron annihilations.

We can describe the potential binding a quark-antiquark pair as $V c=-(4 / 3) \alpha S(r) \hbar c / r+k r$, where $\alpha S$ is the coupling constant between quark and gluon, similar to the fine-structure coupling seen in electromagnetism $(\$ 6)$ or to the constant $\alpha W$ associated to the weak force ( $(8)$. As $\alpha S \sim 1$, we have $\alpha S / \alpha E M \sim 100$ and $\alpha S / \alpha W \sim 10^{5}$, hence the name 'strong' interaction. These values also justify why the perturbative theory ( $\$$ 6) doesn't apply. The first term appearing in $V c$ is the usual Coulomb potential, and is due to colour charges exchanging massless gluons. The second summand specifies the colour's force: being proportional to $r$ it is small at close distances, whence $V c(r \rightarrow 0) \sim 1 / r$. However, $V c$ doesn't diverge when $r \rightarrow 0$ because $\alpha S(r)$ isn't constant; rather, it decreases as distances get smaller. This feature, typical of QCD, goes under the name of asymptotic freedom: the potential is quite weak for two very close quarks, which thus behave as if they were free particles. As $r$ increases the potential strengthens and generates a force similar to that of an elastic band: $V c(r \rightarrow \infty) \rightarrow \infty .^{6}$ This is the characteristic property that confines quarks and gluons to remain inside hadrons and "condemns" them to be imprisoned inside. Quarks and gluons cannot escape and be observed directly like ordinary free particles: the colour charge cannot be observed. If we try to free quarks by stretching the "gluey elastic band" that ties them together, the elastic band breaks and a quarkantiquark pair is created. At extremely high temperatures $\left(>10^{18} \mathrm{~K}\right)$ the hadronic matter is believed to undergo deconfinement: this is a proper phase transition, whereby isolated colour charges, which in a "colder" ambient would move within a tenth of a fermi, can now freely roam the entire "hot" region. This is a sign of how our vision of the world strongly depends on the energy scale at which we live.

We may view the interaction of two nucleons as a manifestation of the "residual" strong interaction between colour charges. The pion-nucleon interaction investigated between the 1940s-1950s is not a fundamental interaction but simply a residual effect of the strong force binding quarks inside nucleons and mesons. Nuclei are kept together by the residue colour force between nucleons (which are neutral, on average, with respect to the colour charge). Positively charged nuclei and electrons are bound by the electromagnetic force and form atoms that are normally electrically neutral. The residual electromagnetic force between atoms gives rise to the bonds that tie atoms inside a molecule together. Strong forces weaken very rapidly as distances increase, and become negligible between two extremely close nuclei, for example inside a molecule, since an atom $\left(\sim 10^{-8} \mathrm{~cm}\right)$ is much bigger than a nucleus $\left(\sim 10^{-13} \mathrm{~cm}\right)$. The limited range of nuclear forces is the reason why all physical and chemical properties of solid bodies are determined exclusively by the electromagnetic interactions of their electrons.

QCD is a consolidated theory: it has been confirmed by convincing experiments over the past 40 years, and has spawned a corpus of experimental data resting on the idea that quarks and gluons interact by the forces described by QCD.

\section{The electroweak theory}

Is it possible to describe the weak force using a gauge theory, as is the case for the strong and electromagnetic

\footnotetext{
${ }^{6}$ To understand how powerful the strong force is let us assume the constant $k$ appearing in the formula for $V c$ is $1 \mathrm{GeV} / \mathrm{fm}$, i.e., $10^{15}$ $\mathrm{GeV} / \mathrm{m}\left(1 \mathrm{fm}=1\right.$ fermi $\left.=10^{-15} \mathrm{~m}\right)$. Since $1 \mathrm{eV}=1.602 \times 10^{-19} \mathrm{~J}$ and $1 \mathrm{~J}=1 \mathrm{~N} \times 1 \mathrm{~m}$, it follows that $1 \mathrm{GeV} / \mathrm{fm}=0.16 \times 10^{6} \mathrm{~N}$, which means a force of 16 tons! For the studies that lead to the notion of asymptotic freedom Frank Wilczek, David J. Gross and H. David Politzer were awarded the 2004 Nobel Prize.
} 
forces? The answer is yes, but the resulting formulation includes, besides the weak force, the electromagnetic interaction as well, thus providing the two with a common origin: this is the electroweak theory.

We left the theory of the weak force to deal with the repercussions of the discovery that parity is not preserved (1956), culminating in the $V$-A theory (1958). Let us recall the first advancements made within Yang-Mills theory (1954). For the theory to be renormalisable ( $\$ 5$ ) fieldcarrying bosons must be massless. This is exactly what happens for mediator bosons in QED (photons) and QCD (gluons). So what about the weak force? Something seems to be wrong, because weak interactions have a short range, and as such (see $\S 3$ ) they must be mediated by bosons with a mass. But massive bosons break the gauge invariance, so we seem to have fallen into a deadlock. The solution came from the notion of spontaneous symmetry break, introduced in particle physics at the beginning of the 1960s by Yōichirō Nambu (Nobel Prize in 2008) and Giovanni JonaLasinio. Thanks to the process of spontaneous break of the symmetry it was possible to endow the gauge bosons of the electroweak theory with a mass, and also come up with a mechanism to confer a mass to all known particles.

In order to illustrate the concept let's consider a magnet. At room temperature its magnetic dipoles are all coherently aligned. If the magnet's temperature rises above a given threshold (called Curie temperature) it loses its ferromagnetic properties and does not attract iron objects, because the dipoles are now oriented randomly. There's no longer a preferred spatial direction, and the system is invariant under space rotations. If we decrease the temperature below the Curie point, the dipoles realign once again. The system hasn't changed but below the Curie point it doesn't possess the symmetry it had above the critical temperature: the symmetry under space rotations has been broken spontaneously by raising the temperature above the Curie point. ${ }^{7}$ Weak interactions can be described in terms of spontaneously broken gauge symmetries. What causes such symmetry breaks? At this point the Higgs boson enters the stage.

In 1961 the British physicist Jeffrey Goldstone considered a complex-valued scalar field $\varphi$ (mapping points in space to pairs of real numbers) and a potential function of the form $V H=\mu^{2} \varphi^{*} \varphi+\lambda\left(\varphi^{*} \varphi\right)^{2}$, with $\mu, \lambda$ constants and $\varphi^{*}$ denoting the complex-conjugate of $\varphi . V H$ is nowadays known as the Higgs potential. The system considered by

\footnotetext{
7 Abdus Salam gave the following example of symmetry break. Suppose $N$ people sit at a round table and there are $N$ sandwiches halfway between any two individuals. Each person has one sandwich on the right and one on the left, at the same distance. For the lunch to start one person must break the symmetry of the layout by choosing a sandwich (the one at his left, for instance), at which point everybody else is forced to do the same.
}

a
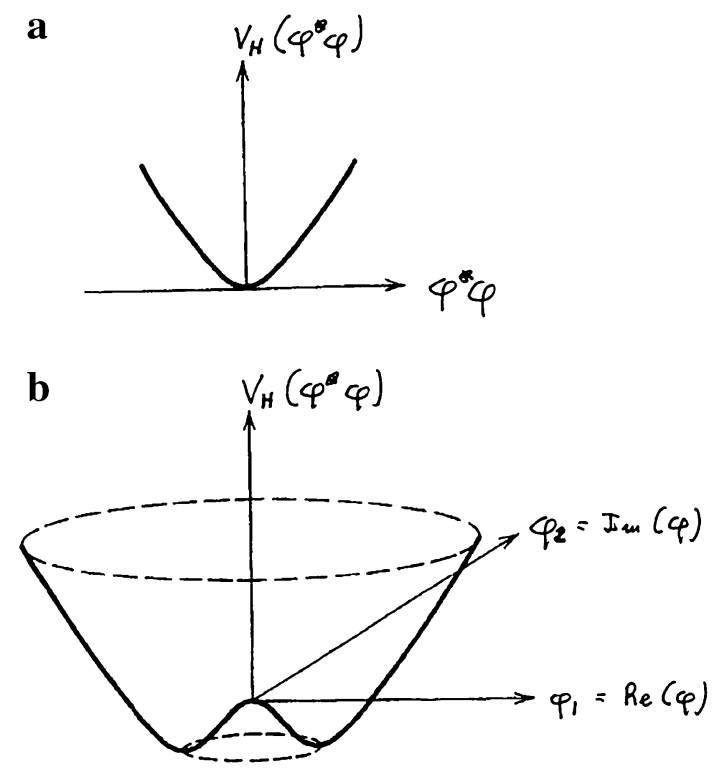

Fig. 2 The potential $V H=\mu^{2} \varphi^{*} \varphi+\lambda\left(\varphi^{*} \varphi\right)^{2}$ in cases (a, above) $\mu^{2}>0$ (bowl-shaped) and (b, below) $\mu^{2}<0$ (sombrero) (Source: adaptation from R. Eisberg, R. Resnick, Quantum Physics of Atoms, Molecules, Solids, Nuclei and Particles, Wiley, New York, 1985)

Goldstone was invariant under a global gauge transformation like $\varphi \rightarrow \varphi^{\prime}=\mathrm{e}^{-\mathrm{i} \alpha} \varphi$. In the case $\mu^{2}>0$ the potential is shown in Fig. 2a. It's clear that the potential is symmetric. If we decrease $\mu^{2}$ we reach a critical point (think of the Curie point) corresponding to $\mu^{2}=0$. In fact, for $\mu^{2}<0$ (when $\mu$ is imaginary), the potential (Fig. 2b) remains symmetric but changes shape, and looks like the bottom of a champagne bottle or a sombrero. The bowl shape (Fig. 2a) is perfectly symmetric about its axis, and the same is true for the sombrero, although the latter may present spontaneous symmetry breaking.

In the convex-shaped case a ball placed inside the bowl always remains in the trough, and if nudged it oscillates regularly around the lowest point, i.e., the equilibrium, irrespectively of the direction it is nudged in, because the bowl's curvature is the same in every direction. In this case the potential $V H$ reaches a minimum at $\varphi=0$, that is, in vacuum. In the other case, too, placing the ball in the middle determines a symmetric situation. But now the state $\varphi=0$ corresponds to a local maximum and hence to an unstable equilibrium. The ball can fall spontaneously from the middle bump to one minimum state for $V H$ (the locus of minimal energy, given in the $\varphi_{1} \varphi_{2}$-plane by the circle centred at the origin with radius $v: \varphi_{1}^{2}+\varphi_{2}^{2}=$ $-\mu^{2} / \lambda=v^{2}$ ): the symmetry is broken since the ball stops at some point on the circle, and the corresponding state is not rotationally invariant. To discuss the case $\mu^{2}<0$ we need to consider what happens at a point on the bottom circle: we may choose any point, but we have to make a choice, which means breaking the system's symmetry. The 
spontaneous break of symmetry is a natural and unforced process whereby a system, initially in a symmetric state, ends up being asymmetric. When this occurs the potential $V H$ reaches its minimum value (that of lowest-possible energy) not in vacuum, i.e., for $\varphi=0$, but, as we say, for a "nonvanishing expected value" $\langle\varphi\rangle=v$. In the lowestenergy state, instead of having $V H=0$ everywhere, $V H$ is constant but nonzero. It's as if the vacuum state was filled with the field's "background noise" at its minimum points. This is an unexpected property of the Higgs field $\varphi$. In physics the state in which fields have lowest energy is called vacuum. When the Higgs field is uniformly constant but different from zero its energy attains the minimum value (among all states where the field is turned on). Hence, in vacuum, the Higgs field does not vanish.

In the case of the sombrero-shaped potential the ball, once in motion, oscillates around the equilibrium point if pushed up the slope, and doesn't oscillate if pushed towards the trough because there is no resistance to the motion. Well, what Goldstone found is that in correspondence of symmetry breaking for radially-symmetric motions a massive particle appears (the mass is due to the energy required to overcome the ball's inertia), and for motions along the circle of minima a massless particle appears (no resistance to motion here). Both particles were called "Goldstone bosons".

In 1964 the British physicist Peter W. Higgs studied a complex-valued scalar field in presence of local gauge symmetry. He considered a local phase transformation (§ 7) that required the existence of a particle carrying the interaction: the gauge boson (which in electromagnetism, as we saw, is identified with the photon). Using the potential of Fig. 2b Higgs obtained two particles from the spontaneous break of the symmetry, one with mass and one without. And here's the surprise: by a suitable gauge transformation the Goldstone boson disappeared from the theory and the gauge boson acquired a mass! It looked as if the gauge boson had "eaten" Goldstone's boson thus becoming "heavy". This is the so-called "Higgs mechanism", which reconciles the gauge invariance with the existence of massive gauge mediators, although at the price of introducing a new interaction. The zero-mass particle that originated from spontaneous symmetry breaking - the Goldstone boson-turned into a property of the gauge boson, while the massive particle corresponding to the field's fluctuations outside the minimum locus is the socalled Higgs boson. ${ }^{8}$

\footnotetext{
${ }^{8}$ What does vacuum look like? According to quantum mechanics and the uncertainty principle a vacuum is not really 'empty', but it oscillates constantly between an absolutely empty state and a state containing particle-antiparticle couples. Recall what we said in $\S 3$ : by virtue of $\Delta E \Delta t \geq \hbar$, in order to measure the energy $\Delta E$ we need at least $\Delta t$ time, and within a $\Delta t$ lapse we cannot "detect" the lack of
}

In a gauge theory the mediators of the force, like the electromagnetic field's photon, should be massless in order to guarantee the symmetry they enjoy. For a spontaneous break of the symmetry, though, the propagation of the gauge field in a vacuum filled by the "background noise" of the Higgs field generates a mass, due to the interaction with the Higgs itself. A frequent analogy used to explain this mysterious "Higgs mechanism" is that of a congress of scientists (the Higgs field) at which a big shot shows up (the massless gauge boson). As a result of the pushing and shoving of people trying to approach him, the luminary walks around in the room with difficulty; since he cannot move at the speed of light anymore (the price of fame), he gains inertia, i.e., mass. What makes the gauge field massive is the Higgs's anomalous vacuum property of having a nonvanishing expected value $v$.

The prototype of gauge electroweak theory, in which electromagnetism and weak forces are coalesced, was elaborated in 1961 by Sheldon L. Glashow, although he didn't manage to endow bosons with a mass. In 1967-68 Steven Weinberg and Abdus Salam, by applying the Higgs mechanism, independently managed to give the gauge bosons of the electroweak theory a mass. In 1971 the Dutch Gerardus t'Hooft proved the theory was renormalisable. At that point all ingredients were present: the electroweak theory of Glashow-Weinberg-Salam (1979 Nobel laureates) and the proof of its renormalisability by t'Hooft (Nobel Prize in 1999, shared with his teacher and colleague Martinus J. G. Veltman) became the foundations for understanding the weak interaction, which people had been working on since the late 1950s.

Before we continue let us describe another family of particles that we know, besides quarks: leptons (from the Greek $\lambda \varepsilon \pi \tau o ́ s:$ delicate, light, small). Scattering experiments have proved that the size of quarks and leptons is less than $10^{-18} \mathrm{~m}$ : they truly behave like point-particles. In

Footnote 8 continued

$\mathrm{D} E$. In vacuum all possible particle-antiparticle couples can be created and annihilated so quickly that any measurement becomes impossible. In certain situations, though, the effects of these fluctuations in vacuum can be seen. An electron in vacuum can emit virtual photons, which in turn can emit virtual couples $e^{+} e^{-}$. These pairs affect the dielectric constant in vacuum, and hence Coulomb's law changes at very small distances with respect to $\hbar / m e c=3,8 \times 10^{-13}$ $\mathrm{m}$ ( $m e$ denotes the mass of the electron). Because of the complicated structure of vacuum, therefore, at very close range the electromagnetic force is stronger than what the Coulomb law prescribes. It is believed that also the Higgs mechanism should be reducible to this pattern. The Higgs field interacts so intensely with itself that its average value in vacuum is very large (around $180 \mathrm{GeV} / \mathrm{c}^{2}$ ).

Let us remark, to conclude, that even if the name Higgs is the only one that is always cited, many scholars developed independently, and almost simultaneously similar ideas: the Belgians François Englert and Robert Brout (1964), the Americans Gerald S. Guralnik and Carl R. Hagen and the British Thomas W. B. Kibble (1964). 
comparison, protons are of the order of $10^{-15} \mathrm{~m}$. No excited state of a quark or lepton has been observed so far, so they do seem to be elementary particles. At the moment we know six kinds of leptons and six of quarks (together with their antiparticles). Quarks and leptons have semiinteger spin $(1 / 2)$ so they belong to fermions. The leptons are the electron $e$, the muon $\mu$ and the tau particle $\tau$, plus the corresponding electron, muon and tau neutrino $v e, v_{\mu}$, $\nu_{\tau} .{ }^{9}$ Leptons can be organised in three subfamilies: the electronic family $(e, v e)$, the muon family $\left(\mu, v_{\mu}\right)$ and the tau family $\left(\tau, v_{\tau}\right)$. Similarly to the baryon number we can define lepton numbers $L e, L_{\mu}, L_{\tau}$, for each subfamily or 'flavour', which are preserved independently. Their sum gives the total lepton number $\mathrm{L}$.

Apart from endowing gauge bosons with a mass, the interaction with the Higgs field explains how the known fermions (quarks and leptons) acquire their mass. This mass is proportional (the factors are the 'Yukawa constants') to the force with which the Higgs field interacts with each fermion. To sum up, the particles of the Higgs field generate the mass of: the Higgs boson, by means of the self-interacting force; the carriers of the electroweak force, by their interaction with the Higgs field; all massive particles, by means of "ad hoc" forces in each case. Clearly this doesn't explain why, for instance, the electron has a mass: we have merely substituted the mass issue with a different, equally impenetrable mystery: the coupling between the electron and the Higgs field (whose mass the theory doesn't predict).

At the level of quarks and leptons, which we may consider the ultimate constituents of matter, the weak interaction occurs between two quarks, two leptons or one quark and one lepton. Quarks and leptons therefore carry a weak charge. Neutrinos are subjected only to weak interactions; charged leptons $(e, \mu, \tau)$ feel the weak force and the electromagnetic one. The weak force doesn't preserve certain quantities (such as parity, charge conjugation and strangeness) that are instead preserved by the strong and electromagnetic interactions. We saw that the weak force is less intense than both the strong and the electromagnetic force, and unless the latter two do not intervene due to some conservation law, it typically gets eclipsed by them.

The electroweak theory is a renormalisable non-Abelian gauge theory governed by the symmetry group $\mathrm{SU}(2) w \times \mathrm{U}(1) Y w$. $\mathrm{SU}(2) w$ is the weak isospin group: despite the name it has only a very loose relationship to the

\footnotetext{
9 That the electron and tau neutrinos are indeed distinct particles, as conjectured by Bruno Pontecorvo in 1960, was confirmed in a 1962 experiment by Fermi's student Jack Steinberger, Leon Lederman and Melvin Schwartz (Nobel Prize in 1988). The lepton $\tau$ (tauon) was detected during a series of tests between 1974 and 1977 by Martin Lewis Perl (1995 Nobel Prize) and his collaborators, while the existence of the tau neutrino was announced officially in 2000 .
}

isospin of strong interactions. From the point of view of Yang-Mills gauge theory, though, the weak isospin renders the couple (ve,e) $L$ equivalent to the proton-neutron couple $(p, n)$ encountered when discussing the strong force. We can similarly treat the other lepton families $\left(v_{\mu}, \mu\right) L$ and $\left(v_{\tau}, \tau\right) L$. The charge of the weak force is the third component $I_{3} w$ of the weak isospin vector. The subscript $L$ (left) indicates that we are considering left-handed particles, since the weak force mediated by charged bosons acts only on particles with left-handed helicity, i.e., those whose spin is opposite to the direction of motion $(\$ 8)$. For this reason the weak isospin's third component is set to $\pm 1 / 2$ for left-handed particles, while right-handed particles (like $e R, \mu R, \tau R$ ) are singlets for the weak isospin. It is assumed that the neutrino has no mass (even if recent experiments seem to indicate that they do have a tiny, nonzero mass, see $\S 12$ ), whence right-handed neutrinos cannot exist. Yet right-handed particles such as the ones mentioned do exist, and the overall theory must deal with them: electromagnetism, for instance, treats both helicities equally. For these reasons the symmetry group $\mathrm{U}(1) Y w$ is introduced into the theory, just as in electromagnetism. $Y w$ is the weak hypercharge, equal to 1 and defined by $Q=I 3 w+(1 / 2) Y w$.

Four gauge fields and four gauge bosons carry the weak force. Three form a triplet with $I w=1$ for the group $\mathrm{SU}(2) w$, and one comes from U(1)Yw. Before the symmetry breaks the bosons are massless. Electromagnetic interactions originate either in the exchange of the neutral boson in the $\mathrm{SU}(2) w$-triplet, or in the $\mathrm{U}(1) Y w$ one, and therefore the charge of this group cannot coincide with the electric charge. The two neutral fields observed experimentally (the photon $\gamma$ and the boson $Z^{0}$ ) are a linear combination of the above two neutral gauge bosons that is governed by a mixing parameter $\theta W$ called "Weinberg angle". The coupling constants ("charges") with the massless gauge bosons of $\mathrm{SU}(2) w$ and $\mathrm{U}(1) Y w$ are denoted by $g$ (in analogy to the electric charge $e$ ) and $g^{\prime}$. Under spontaneous symmetry breaking the initial four gauge bosons, by interacting with the Higgs field, give rise to three massive bosons (combinations of the states of the massless gauge bosons): two charges ones $\left(W^{ \pm}\right)$and one neutral $\left(Z^{0}\right)$. The last real boson carrying the electroweak force is the photon $\gamma$, which we know remains massless and with spin 1. After the symmetry has broken, the coupling charges with the massive bosons and the photon transform into the weak charge $g W$ and the electric charge $e$. The real matter fields $\gamma, W^{ \pm}$, and $Z^{0}$, hence, arise from the massless gauge fields after symmetry breaking.

The simplest way to endow the electroweak bosons with a mass is to replace the complex field $\varphi$ seen earlier with a doublet $\left(\varphi^{+}, \varphi^{0}\right)$ of complex fields, i.e., four Higgs scalar fields, two charged and two neutral. The charged fields give 
mass to the bosons $W^{ \pm}$, one of the neutral fields gives mass to the neutral boson $Z^{0}$, and all three vanish under suitable gauge transformations. The remaining neutral particle $H^{0}$ is the "Higgs boson" and the focus of current intensive studies.

The prediction of the existence of a neutral boson $Z^{0}$ was unexpected. This boson should mediate weak processes called weak-current interactions, which had never been observed previously. These interactions (an example: $v_{\mu}+p \rightarrow v_{\mu}+p$ ) were discovered for the first time at CERN in 1973, thus establishing the first triumph of the electroweak theory and raising a tough question at the same time.

To understand its impact, let's go back to the notion of universality of weak interactions $(\S 8)$. The processes studied at the end of the 1940s by Puppi et al. were of the following sort (in modern notation: we employ the symbols of neutrinos that were discovered only later):

(1) beta radioactivity: $n \rightarrow p+$ and $+\bar{v}_{e}$

(2) muon decay: $\mu^{-} \rightarrow$ and $+\bar{v}_{e}+\mathrm{v}_{\mu}$

(3) muon capture by a nucleus: $\mu^{-}+p \rightarrow n+\mathrm{v}_{\mu}$

With the data available in the 1950s these processes seemed to be characterised by the same coupling values. This suggested the idea that weak interactions were universal. At the beginning of the 1960s, with the publication of the data on the decay of strange particles, things became more complicated; one example is the.

(4) decay of the hyperon $\Lambda: \Lambda \rightarrow p+$ and $+\bar{v}_{e}$.

In the latter formula, albeit very similar to the neutron's decay (1), the intensity of the weak force is much lower (one-fifth) than in beta radioactivity. Moreover, increasingly precise data showed that the weak forces in processes (1) and (2) differed by a few percentage points.

These results were eventually explained by Nicola Cabibbo in 1963, before the introduction of quarks. Let's run through his argument in modern language. Up to that point it was known that leptons were eigenstates of the weak force and quarks eigenstates of the strong force. Cabibbo's line of thought would later allow to understand that quarks are eigenstates of the weak force as well, but that the involved quarks are not those with flavour $u, d, s$, typical of the strong force, but rather a linear combination thereof, which therefore depends on a rotation $\theta c$ (the Cabibbo angle). Consequently, the mass eigenstates of the quarks $u, d, c$ are not the same as the weak eigenstates $u c$, $d c, c c$. The entities we call quarks are seen differently by the weak force. It follows that the apparently different values of the coupling constant depend on the mixture of quarks, because the weak eigenstates differ from the strong eigenstates. Measures for the Cabibbo angles have given $\theta c=0.235 \mathrm{rad}=13.5^{\circ}$. Returning to the previous examples, we will say that in the first three the strangeness doesn't change $(\Delta S=0)$, whereas in the last case $\Delta S=1$. In process (1) the coupling constant is $G^{2} F \cos ^{2} \theta c$ while in (4) it equals $G^{2} F \sin ^{2} \theta c$. Since $\sin \theta c=0.235$ and $\cos \theta c=0.972$, any process with $\Delta S=0$ has an effective coupling constant that is larger than in situations where $\Delta S=1$. The consequence for quarks is that $s$ cannot be coupled with the boson $W^{+}$as strongly as $u$ can. Hence the quark doublet seen in weak interactions is not $(u, d)$ but $(u$, $d c)=(u, d \cos \theta c+s \sin \theta c)$.

This brings us to the issue raised by weak neutral currents. If $Z^{0}$ interacts with the quark $u$ there is no problem; but if $Z^{0}$ interacts with $d c$, we have to remember the latter is a mixture of $d$ and $s$. This would give neutral-current processes in which strangeness varies, a phenomenon never observed previously. In 1970 Glashow, John Iliopulos and Luciano Maiani found a way out: if there existed a fourth quark $c$ (recall: at that time only $u, d$ and $s$ were known), then there would be another doublet $(c, s c)=(c, s \cos \theta c-$ $d \sin \theta c$ ) able to cancel the processes $s \rightarrow d$ where strangeness varied. The experimental detection of the hypothetical particle $c$ in 1974 represented a big success for the electroweak theory and the GIM mechanism, thus called after its creators.

The theory of mixtures was extended to include two more quarks $(t$ and $b)$, and 1972 saw its codification in a $3 \times 3$ matrix by Makoto Kobayashi and Toshihide Maskawa (2008 Nobel Prize). ${ }^{10}$ The basic assumption has remained the same: there is no neutral-current process (i.e., mediated by the boson $Z^{0}$ ) that changes the flavour of quarks.

The massive bosons $W^{ \pm}$and $Z^{0}$ were first observed in 1983 during an experiment at CERN carried out by Carlo Rubbia and Simon Van der Meer (1984 Nobel Prize). They had the "correct" mass predicted by the theory, that is, $M W=M Z \cos \theta w=\left(e^{2} \sqrt{ } 2 / 8 G F \sin ^{2} \theta w\right)^{1 / 2}$. This means the values of the masses of $W^{ \pm}$and $Z^{0}$ are related, and depend on the intensity of the electromagnetic force $(e)$, the weak force $(G F)$ and also their mixture, through $\theta w$. This angle is one of three parameters that the theory requires as experimental inputs (the choice is not unique, for any one among $e, G F, \theta w$ will do), and that can be measured in several independent tests: the comparative analysis has given $\sin ^{2} \theta w=0.2319$. The experiments have also produced $M W=80.2$ and $M Z=91.2 \mathrm{GeV} / c^{2}$, closely matching theoretical predictions. ${ }^{11}$

\footnotetext{
10 The so-called CKM matrix, thus called after the authors of the mixture theory (Cabibbo, Kobayashi and Maskawa).

11 Note that these values are rather large for fundamental particles, similar to the mass of medium-sized nuclei as in rubidium and molybdenum.
} 
The photon is the unique real boson without a mass among those carrying the electroweak force. The electroweak theory subsumes the whole QED, as predicted; what is new is the relationship between the electric charge $e$ and the weak charge $g W$ determining the intensity of the corresponding forces: $e=2 \sqrt{ } 2 g W \sin \theta w$. The formula reveals that the two forces are comparable: what renders the weak force so 'feeble' are the large masses $M W$ and $M Z$, which in turn make its range $R$ small ( $R$ is, as a matter of fact, related to the mass $m$ of the carrier particle by $R=\hbar / m c$ ). This fact can be seen directly from the relationship between Fermi's coupling constant and the weak charge: $G F=\sqrt{ } 2 g^{2} W / 8 M^{2} w$. There are further constraints among the coupling "charges" $g$ and $g^{\prime}$ (introduced before symmetry breaking), $\theta w$ and $e$, namely: $\operatorname{tg} \theta w=g^{\prime} / g$; $e=g \sin \theta w=g^{\prime} \cos \theta w$. The latter two are called unification equations, in that they emphasize the combined aspect of the electromagnetic and weak forces.

Translated from the Italian by Simon George Chiossi.

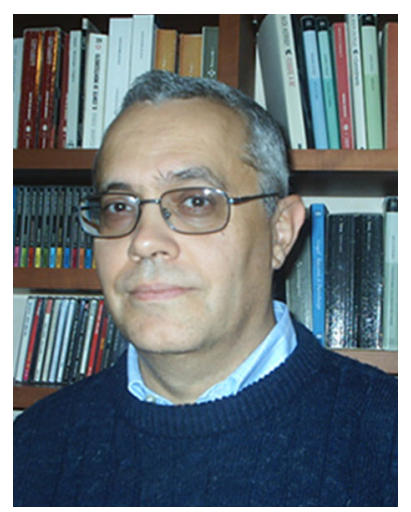

Giulio Maltese has been occupied for a number of years with the history of physics. His research has concerned the history of rational mechanics, relativity and twentieth-century physics in Italy, with a particular focus on Fermi and the group of physicists in Via Panisperna. 